\title{
The Road Map to Preparedness: A Competency-Based Approach to All-Hazards Emergency Readiness Training for the Public Health Workforce
}

Cindy L. Parker, MD, MPH

DANiEl J. BARnett, MD, MPH

Ayanna L. Fews, MBA ${ }^{a}$

DAVID BLODgETT, MD, MPH ${ }^{\mathrm{a}}$

Jonathan M. Links, PhD

\section{SYNOPSIS}

Facing limited time and budgetary resources, state and local health departments need a practical, competency-based training approach to meet the all-hazards readiness requirements of their employees. The Road Map to Preparedness is a training tool designed to assist health departments in providing comprehensive, agency-tailored readiness instruction to their employees. This tool uses an incentive-based, game-like, experiential learning approach to meet the Centers for Disease Control and Prevention's nine core competencies for all public health workers while facilitating public health employees' understanding and acceptance of their emergency response roles. A corresponding evaluation tool, the Road Map to Preparedness Evaluation, yields metrically-driven assessments of public health employee readiness competencies.

Since its pilot in 2003, the Road Map to Preparedness has met with enthusiastic response from participating health departments in the mid-Atlantic region. In addition to its public health impact, the Road Map offers future promise as a tool to assist organizational emergency response training in private sector and nonpublic health first-responder agency settings. 
Public health has adopted unprecedented roles and partnerships in emergency preparedness and response since the terrorist attacks of 2001 in the United States. Consistent with the federal government's move toward an all-hazards model of public health emergency preparedness, local health department workers must embrace a culture of emergency readiness for a broad spectrum of intentional and naturally occurring emergencies.

Achieving this readiness culture requires establishing $24 / 7$ agency response capacity, developing employee awareness of current and emerging large-scale community threats, and fostering a core sense of employees' professional roles as vanguards of public safety and well-being. This mission poses a considerable challenge for a public health workforce historically accustomed to a 9-to- 5 mode of operation and traditionally unfamiliar with the all-hazards approach to readiness demanded in a post-9/11 world.

Faced with limited training budgets, time, and personnel backfill resources, health department employees need an efficient and highly practical educational approach that effectively addresses the Centers for Disease Control and Prevention's (CDC) core readiness competencies for all public health workers. ${ }^{1}$ Within this competency-based framework, preparedness education should also be flexible enough to accommodate the unique response demands and vulnerabilities of individual health departments.

Experience in previous public health emergencies and discussions with public health workers suggest that many public health workers are unlikely to report to duty in response to a mass casualty event. The reasons for this-real to them-include a fear for personal and family safety in emergencies coupled with a lack of insight into how valuable they are to the response effort in times of crisis.

These observations are consistent with research on emergency response perspectives among public health workers. A 2002 study of school health nurses from the New York City Department of Health revealed that $90 \%$ of these nurses reported at least one perceived barrier to reporting to work during an emergency; in order of perceived importance, the top three barriers for these nurses were child and elder care obligations, transportation, and personal health issues. ${ }^{2}$ In addition, research on bioterrorism response attitudes among school nurses and physicians reveals that as the perception of personal risk increases, these healthcare providers are less willing to place themselves at risk to care for patients. ${ }^{3,4}$

An effective public health preparedness training program must address these perceived response barriers, respect the time constraints of a busy public health workforce, and incorporate an educational framework that reflects best practices for adult learning.

\section{ROAD MAP TO PREPAREDNESS: BACKGROUND}

In 2003, Johns Hopkins Center for Public Health Preparedness ${ }^{5}$ began a collaborative training venture with the Montgomery County Department of Health and Human Services Public Health Services (MCPHS) as part of the Project Public Health Ready pilot program sponsored by the National Association of County and City Health Officials (NACCHO). MCPHS set an ambitious goal of training all
600 of their employees in emergency preparedness over a 12-month period.

The Johns Hopkins team worked with MCPHS to develop a training program that would include all nine core competencies developed by the Columbia University School of Nursing and adopted by the CDC for public health workers. ${ }^{1}$

The curriculum needed to accommodate the busy schedules of MCPHS employees through a blend of self-directed and group-oriented trainings. These trainings required an approach that was upbeat, incentive-based, and applicable to daily public health tasks whenever possible to enhance employee acceptance of the training process.

The MCPHS leadership felt that its workforce would respond most successfully to training that incorporated experiential adult learning, a model that makes the student an active participant in the educational process through a "learning by doing" approach. This preference for experiential learning methods is consistent with research on adult education revealing that students' attitudes are significantly more positive about the subject matter in experiential settings than traditional didactic settings. ${ }^{6}$

Besides meeting the nine core competencies, Hopkins and MCPHS desired to implement a culture change of first responder-type emergency readiness for all health department employees. Firefighters and police officers, for example, are well aware that they may be called upon at any time to help protect the public's safety. Public health professionals, on the other hand, are historically not as familiar with the idea that they might be called in to work nontraditional hours in an emergency.

To meet these educational needs and challenges, Johns Hopkins Center for Public Health Preparedness piloted a Road Map to Preparedness curriculum to assist MCPHS in creating and implementing a training plan for all of their employees. Because the work of a busy public health department must continue despite the need for all employees to receive additional training, the complete training program consists of a combination of agency-led activities and selfstudy activities using a game-like, incentive-based model.

\section{ROAD MAP TO PREPAREDNESS: STRUCTURE AND CONTENT}

The Road Map to Preparedness is a series of activities laid out on a game board, with a corresponding curriculum for each activity along the board. ${ }^{7}$ Each employee is given his or her own Road Map Game Board (Figure 1) and Road Map Key (Figure 2), along with a packet of supporting materials. The activities along the board are based on a combination of core public health competencies and the nine core CDC preparedness competencies. In addition to these core competencies, some of the activities included in the Road Map are designed to influence the way public health employees think about their jobs and feel about their role in the agency and about disaster response. The curriculum is designed to require participation in active learning processes while keeping traditional didactic lecture time to a minimum. For example, the Road Map requires employees to map out three alternate routes from home to their designated emergency response locations, accounting for the possibility of unexpected road 
Figure 1. Road Map to Preparedness Game Board

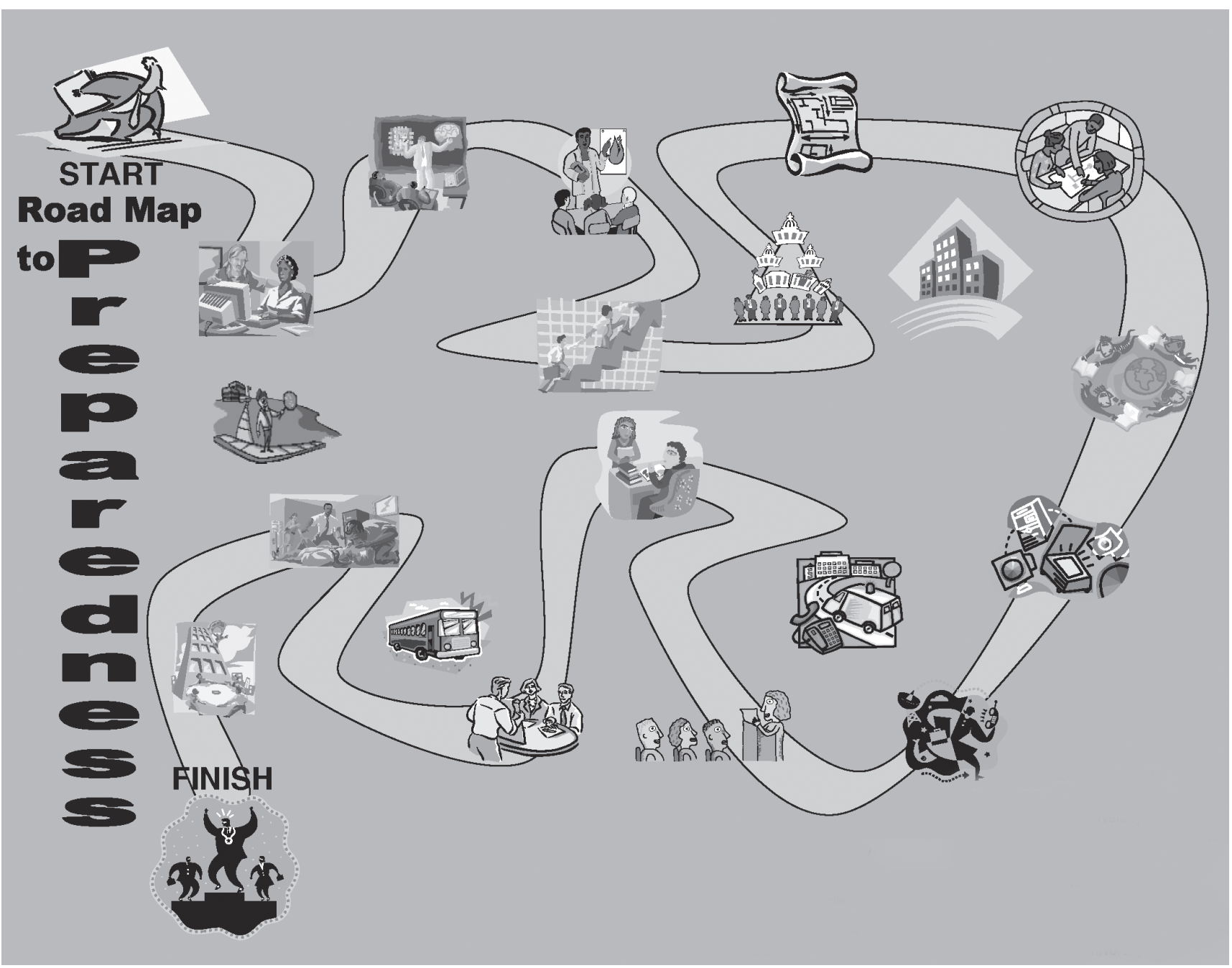

closures. The Road Map is intended to provide an agencywide basic competency level of public health emergency preparedness in the context of ongoing health department-based preparedness trainings and exercises.

Consistent with its incentive-based approach, a series of rewards are offered for employees who successfully complete incremental segments of the Road Map. These rewards are determined by each participating health department and can range from coffee mugs to administrative leave, with larger incentives awarded for faster completion of the Road Map activities.

The first two activities on the Road Map address essential preparedness competencies (Activity 1) and provide an introductory overview of intentional threats to the public's health (Activity 2). Activity 1 requires the participant to complete the Columbia University online preparedness $\operatorname{course}^{7}$ or an equivalent. Activity 2 requires participation in an online or live agency-sponsored training on weapons of mass destruction. These initial activities provide the competency framework and foundational knowledge that partici- pants will need to embark successfully upon subsequent Road Map activities.

Personal and family preparedness must figure centrally in any effective public health workforce preparedness training program. This training should focus on both physical and psychological issues that workers and their families face in a variety of crisis settings, and provide employees with practical tools to deal with these issues. Accordingly, the Road Map places strong emphasis on personal and family safety. Activity 7 requires participants to craft a personal/ family emergency response plan. Health departments are provided the opportunity to offer their participants an agencysponsored lecture or online course on personal/family readiness to provide the necessary background for crafting a personal/family emergency response plan. Personal readiness concepts must be emphasized at work as well as at home, and Activity 4 involves participating in the planning and implementation of a worksite plan to ensure safety and security in an emergency.

An understanding of role-specific response requirements 
Figure 2. Road Map to Preparedness Key

\begin{tabular}{lll}
\hline & Date \\
Completed & Preparedness Activity \\
\hline
\end{tabular}

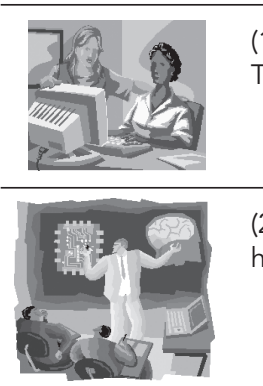

(1) Learn about the public health role in emergency response.

Take the Columbia online course or equivalent. http://cds.osr.columbia.edu/bepcourse/test.asp

(2) Participate in a training about Weapons of Mass Destruction-Online or Agency Sponsored http://www.jhsph.edu/CPHP/Training/Online\%20Training/intro_to_wmd.html

(3) Participate in a training about what your specific primary and alternate roles would be during
an emergency and why it is important to the success of the effort. Identify the limits to your
own authority.
an emergency and why it is important to the success of the effort. Identify the limits to your

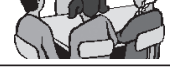

(4) Describe how you are participating in the planning and implementation of a plan at your worksite to ensure the safety and security of staff and clients in the event of an emergency.

(5) Incident Command
Using the templates provided, write your name in the proper place in the chain of command.

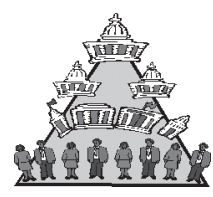

(6) Identify and locate the Health Department's emergency response plan or the section relevant to your role.

(7) Write out your own personal, family emergency response plan using the template provided. Include enough detail that someone else looking at your plan would be able to follow it accurately. Share the plan with your family and everyone involved in carrying out your plan.

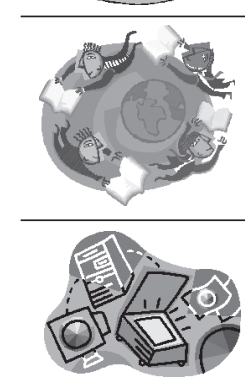

(8) Draw a map showing how you would get from your home to your designated assignment location. Plan 3 different routes in case unexpected street closures obstruct your way.

(9) Demonstrate use of communication equipment. See check-off list provided.

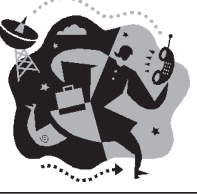

(10) Indicate the ways in which you will be notified in the event of an emergency.

(11) Attend an agency-sponsored lecture on How to Talk to People about Disasters. 
Figure 2 (continued). Road Map to Preparedness Key

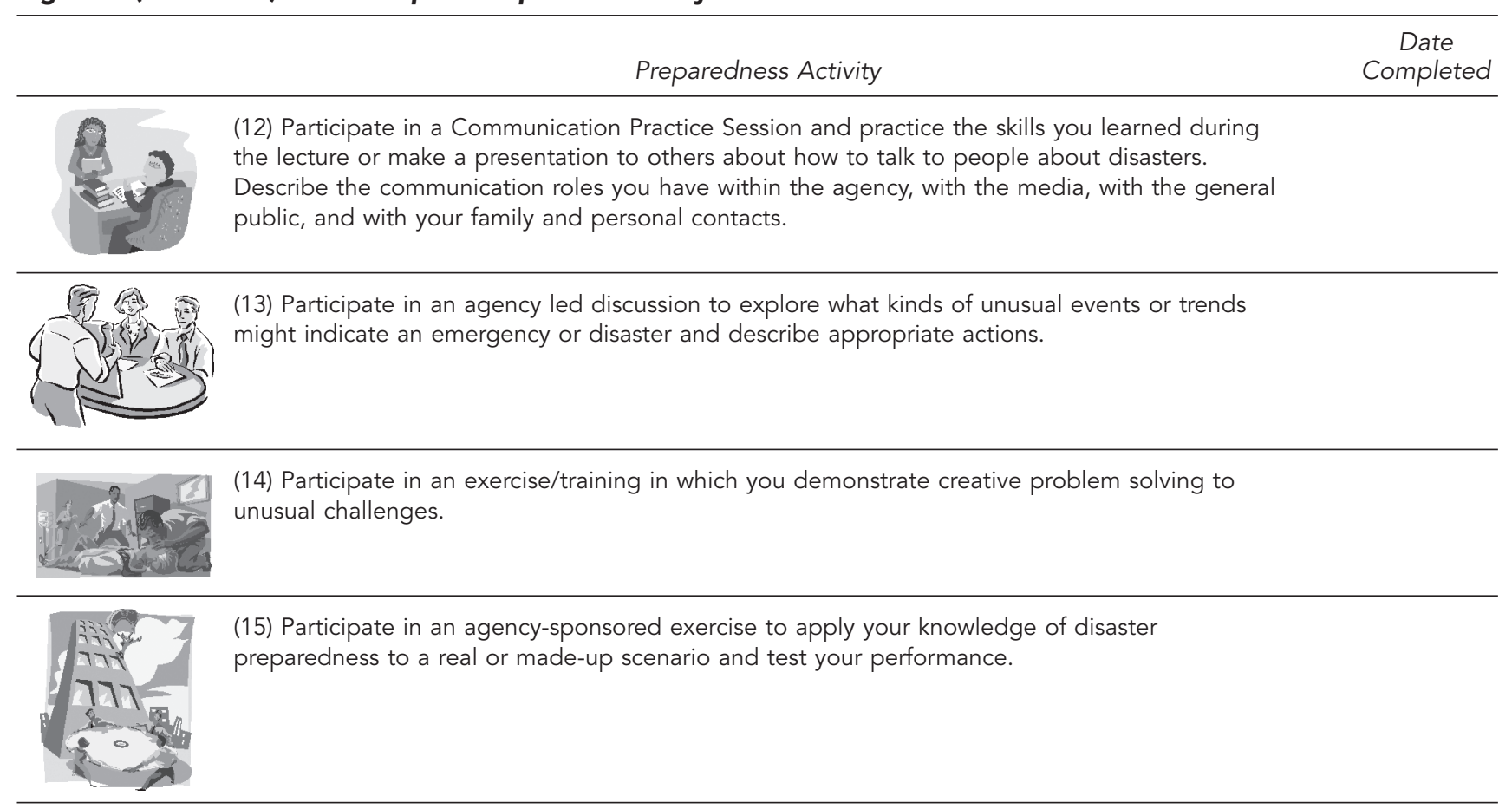

name

has completed all Bioterrorism and Emergency Preparedness activities indicated in this Road Map, or an equivalent demonstrating mastery of the Bioterrorism and Emergency Preparedness competencies.

is essential for effective and swift organizational reaction to a public health crisis. These role-specific learning needs are addressed in Activity 3 (participating in a role-specific training) and Activity 5 (identifying one's place on the health department's incident command chart). These two activities provide each employee with a concrete understanding of the specific role(s) he or she might be asked to perform in a public health emergency. To facilitate universal employee awareness of agency-wide response protocols, Activity 6 asks workers to identify and locate their health department's emergency response plan.

Effective communication skills are at the heart of successful public health readiness and response. Public health workers must not only know how to use different types of communication equipment and how they would be notified to respond to an emergency, but also must understand how to communicate risk and emergency information effectively to the public. Although the media does play an important role in a crisis, every public health worker will likely have a communication role; these can range from answering telephone calls from concerned citizens to talking with community members at dispensing sites or shelters. These communica- tion requirements are addressed in Activity 9 (demonstrate the use of communication equipment per a checklist), Activity 10 (indicate ways in which you will be notified in the event of an emergency), Activity 11 (attend an agency-sponsored lecture on risk communication), and Activity 12 (participate in a communication practice session, describing your communication roles).

From sanitarians to school health nurses, public health workers stand on the front lines of emergency recognition. Therefore, an understanding of individual response roles and agency plans must be firmly coupled with rapid recognition and creative problem-solving skills. Accordingly, Activity 13 requires participation in an agency-led discussion on emergency recognition and appropriate responsive actions; Activity 14 asks employees to participate in a training exercise focusing on creative problem solving. Activities 13 and 14 also serve as team-building activities while they develop vital emergency recognition and response skills.

A culminating agency-sponsored exercise (Activity 15) is an experiential learning activity designed to incorporate the skills and knowledge developed in Activities 1-14. This exercise can range in complexity from a tabletop to a live, full- 
scale exercise, depending on the resources and needs of individual health departments.

The format and content of each activity can be tailored to meet the unique needs of any health department, while still ensuring that each employee successfully completing the Road Map has achieved all nine CDC-adopted "Emergency Preparedness Core Competencies for All Public Health Workers." ${ }^{1}$ For the most part, the activities on the Road Map to Preparedness can be completed in any order. However, the fundamental competency-based framework and knowledge base developed in Activities 1 and 2 provide an ideal starting point, and Activity 15 should be the final activity in the process to develop employees' confidence in their abilities to handle a simulated emergency using the knowledge and skills developed in the preceding 14 activities of the Road Map.

\section{EVALUATION OF THE ROAD MAP TO PREPAREDNESS}

Evaluation of the Road Map, training plan, and curriculum occurs in two ways: (1) each agency-led activity is evaluated by each participant immediately following the activity; (2) the health department completes (and returns to the Center) an evaluation of how the Road Map to Preparedness training plan and curriculum helped the department to prepare for either a real disaster response or an exercise, along with suggestions for improvements to the process.

Evaluation must be an integral part of any training program; to meet this need, the Johns Hopkins Center for Public Health Preparedness has developed a companion Road Map to Preparedness Evaluation tool. As does the Road Map to Preparedness itself, the Road Map to Preparedness Evaluation is competency-based, quantifiable, stepwise, and outcomeoriented.

The Road Map to Preparedness Evaluation generates metrically driven impact assessments-at both the individual employee and organizational levels-for each of the 15 milestones on the Road Map to Preparedness and for the overall effect of the completed Road Map (Figure 3).

These evaluative activities focus on the impact of the Road Map to Preparedness along four major axes: (1) employee knowledge of preparedness concepts; (2) employee familiarity with individual emergency response roles; (3) employee attitudes toward emergency readiness; and (4) organizational efficiency and effectiveness of health department readiness and response as assessed through emergency preparedness exercises.

The Road Map to Preparedness Evaluation uses information obtained from participants immediately after each activity and upon Road Map completion, and from an organizational context once the Road Map is completed. These impact assessments include group baseline performance on pre-tests that are compared to post-test scores immediately after trainings, and subsequently at one-, two-, and threeyear annual intervals. Audience interactive software ${ }^{8}$ allows for onsite electronic administration of pre-tests and posttests on the date of training, as well as for anonymous qualitative feedback on the effectiveness of trainers and relevance of content.
To measure longer-term assessments of the Road Map to Preparedness' employee-level and organizational-level impacts, health departments and their employees will be asked to complete the Road Map to Preparedness Evaluation at one-year intervals for three years following initial completion of the Road Map to Preparedness. An online survey tool called SurveyMonkey could be used for these longer-term evaluations. ${ }^{9}$ These data will serve to direct future trainings within organizations that have already completed the Road Map and will facilitate increased use of the Road Map itself.

\section{NEXT STEPS AND APPLICATIONS}

Since its pilot with MCPHS in 2003, the Road Map to Preparedness has become a centerpiece of Johns Hopkins Center for Public Health Preparedness training efforts with local health departments in Maryland, and has been met with great enthusiasm and success. As of July 2005, Johns Hopkins Center for Public Health Preparedness has implemented the Road Map to Preparedness for eight local health departments in the mid-Atlantic region. At least 20 additional health departments from across the nation have requested the Road Map implementation in 2005-2006.

The Road Map's versatility in meeting the needs of health departments of varying size, location, and resources makes it a widely applicable model for competency-based public health workforce training for all-hazards readiness. Participating health departments have also successfully used the Road Map as a team-building tool by posting a large Road Map Game Board in a central location and keeping track of an agency division's progress, often in friendly competition with other sections or departments. Longer-term evaluation will provide new insights into additional best practice applications of the Road Map to Preparedness.

Ongoing training needs, including staff turnover, will create further opportunities to modify the Road Map. Specific training for more specialized emergency response activities—such as communicable disease epidemiologic response or Strategic National Stockpile management-can easily be added to the Road Map requirements or be addressed as the "Road Beyond" for specific groups of department employees. Other uses of the Road Map, including readiness training for non-public health first responders and for employees in large and small businesses, are currently under development. To the extent that the training needs of local agencies in states beyond Maryland differ significantly, further modifications and extensions are possible. A formal needs assessment in collaboration with practice partners is the best way to identify all opportunities and needs.

The Road Map offers a readiness training approach that is comprehensive, well-received, predictable, and measurable. Through its stepwise, incentive-based, experiential learning methods, employees are able to gain a common language and skill set to aid their participation in readiness drills and their response to actual emergencies.

The development of this manuscript by Johns Hopkins Center for Public Health Preparedness has been supported in part through a cooperative agreement with the Centers for Disease Control and Prevention. 


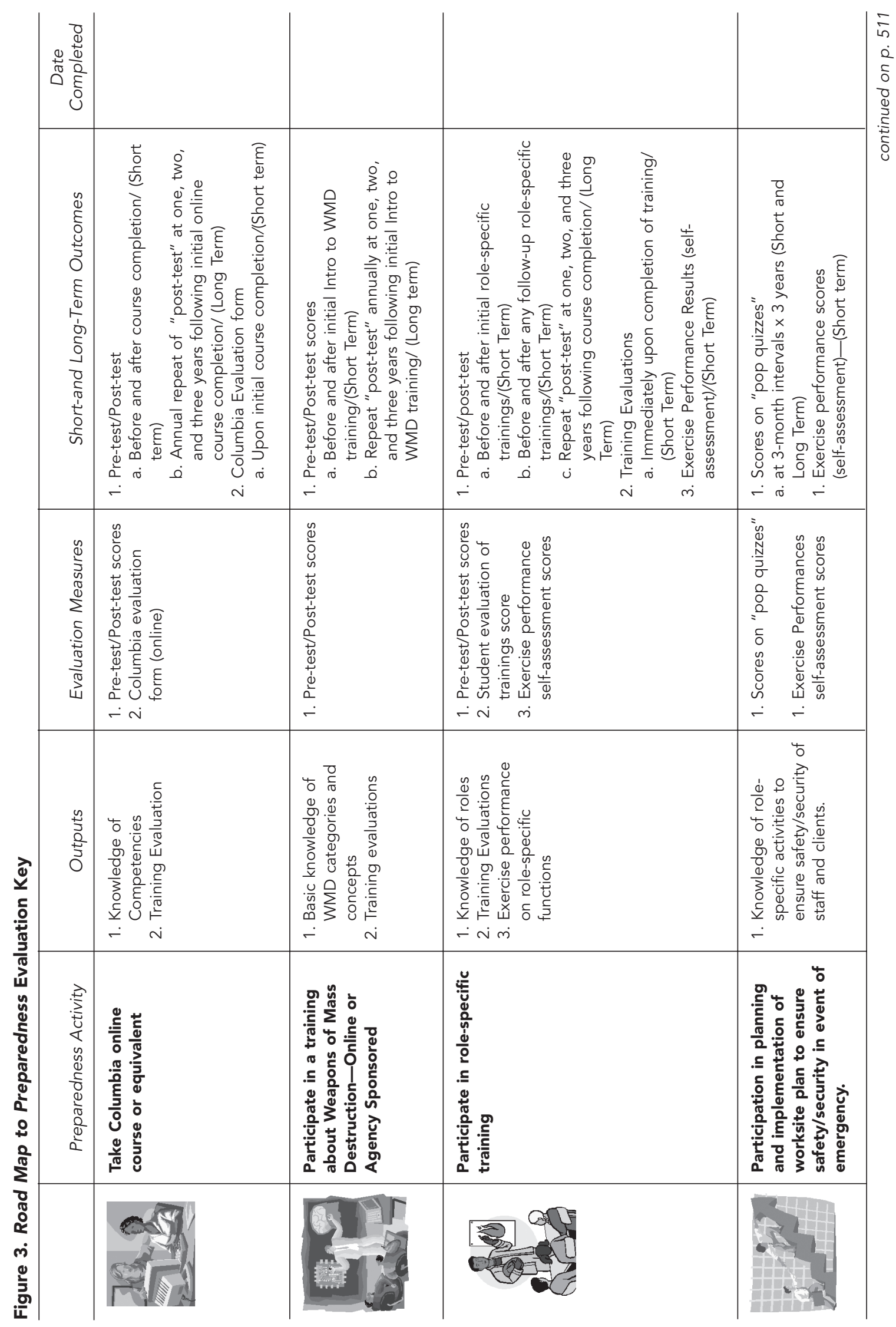

Public Health Reports / September-October 2005 / Volume 120 


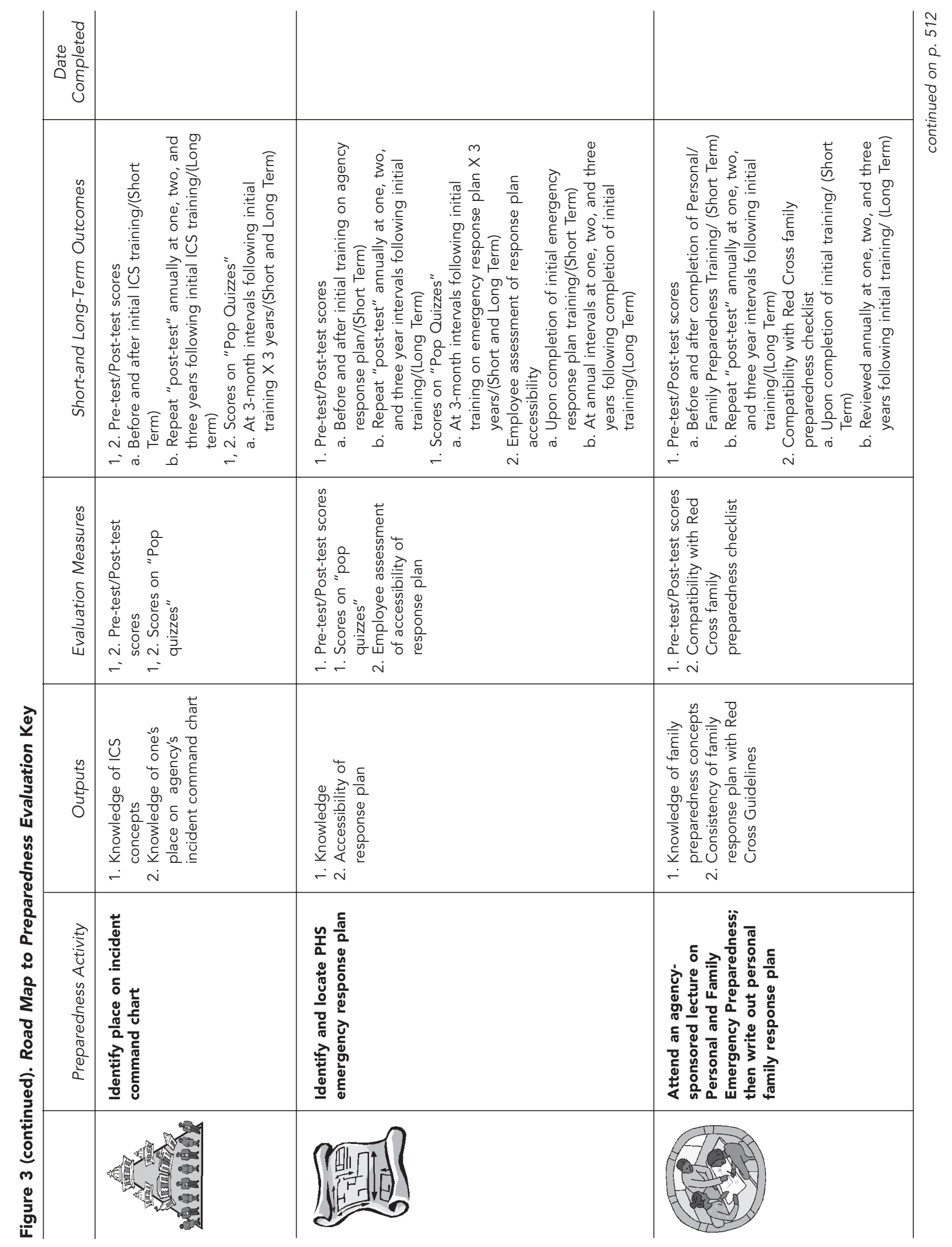

Public Health Reports / September-October 2005 / Volume 120 


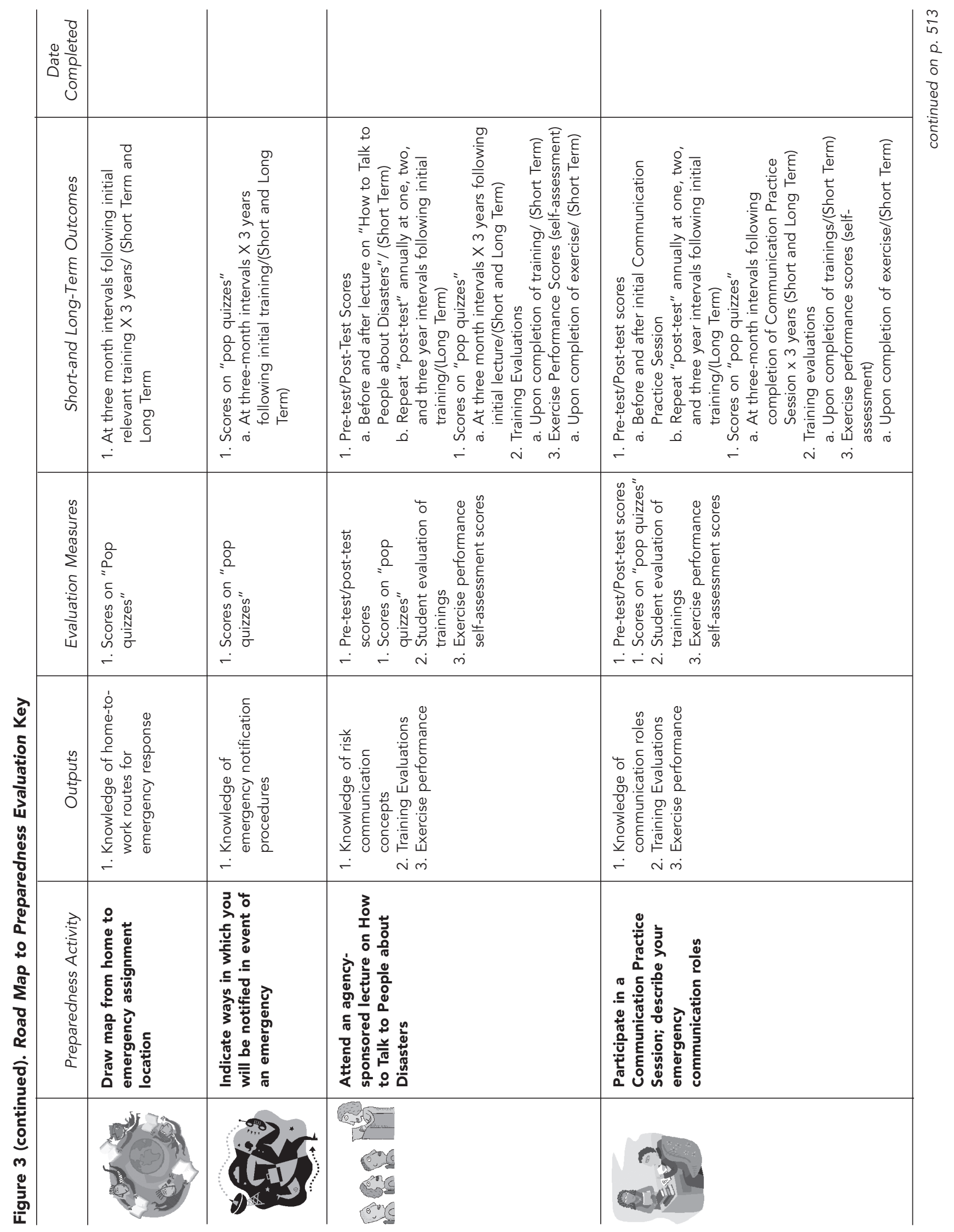

Public Health Reports / September-October 2005 / Volume 120 


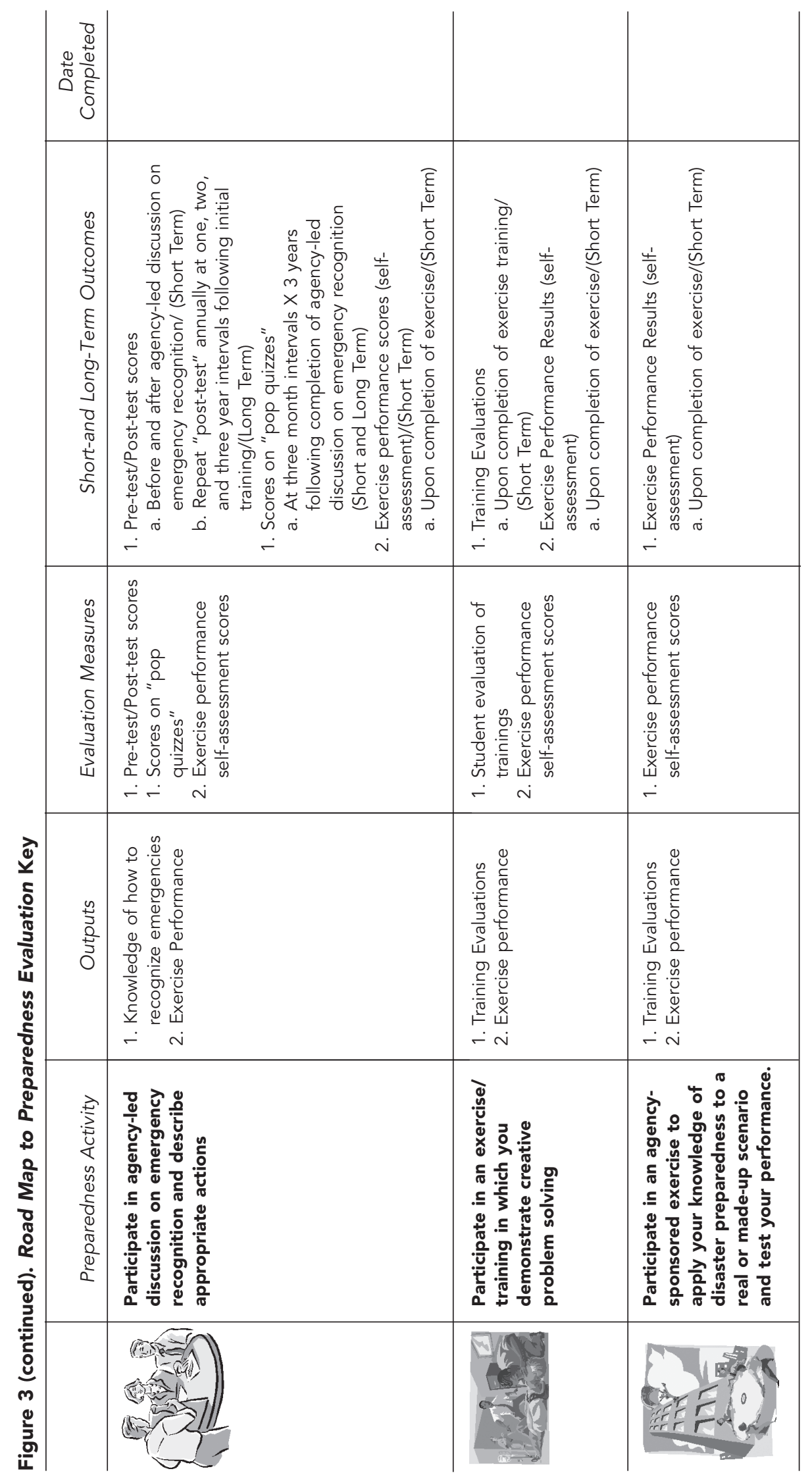

Public Health Reports / September-October 2005 / Volume 120 


\section{REFERENCES}

1. Columbia University School of Nursing Center for Health Policy. Emergency response: core competencies for all public health workers (April 2001) [cited 2005 Jun 8]. Available from: URL: http:// www.cumc.columbia.edu/dept/nursing/institutes-centers/chphsr /btcomps.pdf

2. Qureshi KA, Merrill JA, Gershon RR, Calero-Breckheimer A. Emergency preparedness training for public health nurses: a pilot study. J Urban Health 2002;79(3):415-6.

3. Gullion JS. School nurses as volunteers in a bioterrorism event. Biosecurity and Bioterror 2004;2:112-7.

4. Alexander GC, Wynia MK. Ready and willing? Physicians' sense of preparedness for bioterrorism. Health Aff (Millwood) 2003;22(5): 189-97.
5. Johns Hopkins Center for Public Health Preparedness [cited 2004 Aug 19]. Available from: URL: http://www.jhsph.edu/preparedness/ 6. Pugsley KE, Clayton LH. Traditional lecture or experiential learning: changing student attitudes. J Nurs Educ 2003;42:520-3.

7. Columbia University Mailman School of Public Health. Basic emergency preparedness for all public health workers [cited $2005 \mathrm{Jul}$ 12]. Available from: URL: http://cds.osr.columbia.edu/bepcourse /test.asp

8. eInstruction Corporation. Classroom Performance System: Version 3.5.1.1002. Denton (TX): eInstruction; 2004.

9. SurveyMonkey [cited 2004 Aug 19]. Available from: URL: http:// www.surveymonkey.com/ 